LETTER • OPEN ACCESS

Mutual extinction and transparency of multiple incident light waves

To cite this article: Ad Lagendijk et al 2020 EPL 13034002

View the article online for updates and enhancements.

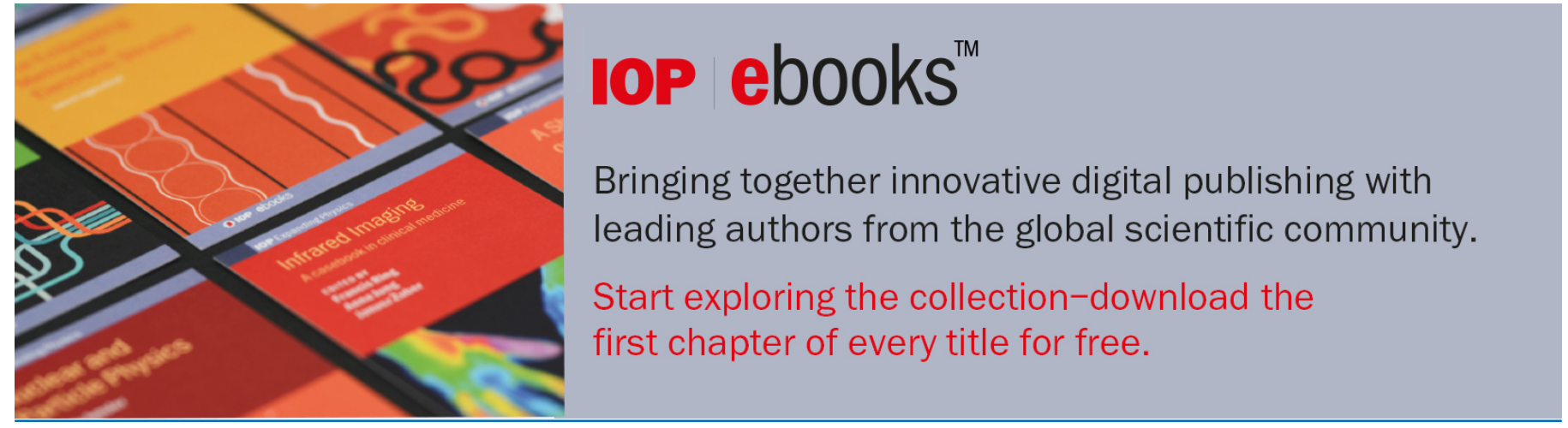

This content was downloaded from IP address 136.143.56.219 on 21/01/2021 at 09:06 


\title{
Mutual extinction and transparency of multiple incident light waves
}

\author{
Ad LagendiJK ${ }^{1}$, Allard P. Mosk $^{2}$ and Willem L. Vos ${ }^{1}$ \\ 1 Complex Photonic Systems (COPS), MESA + Institute for Nanotechnology, University of Twente \\ P.O. Box 217, 7500 AE Enschede, The Netherlands \\ 2 Debye Institute for Nanomaterials Science, Utrecht University - P.O. Box 80000 \\ 3508 TA Utrecht, The Netherlands
}

received 13 May 2020; accepted in final form 19 May 2020

published online 3 June 2020

PACS 42.25.-p - Wave optics

PACS 11.55.-m - S-matrix theory; analytic structure of amplitudes

PACS 03.65. Nk - Scattering theory

\begin{abstract}
When a wave is incident on a complex scattering medium, the transmitted intensity differs from the incident one due to extinction. In the absence of absorption, the extinguished power is equal to the total scattered power, a well-known conservation law termed the optical theorem. Here, we extend the case of a single incident wave to the situation of scattering and extinction by multiple incoming waves. The emerging generalized optical theorem has the exciting consequence that multiple incident waves show mutual extinction and mutual transparency, phenomena that do not exist in common forward scattering or self-extinction. Based on both exact calculations of realistic three-dimensional (3D) samples containing many (up to $10^{4}$ ) scatterers and on approximate Fraunhofer diffraction theory we make the striking observation that the total extinction of two incident waves is greatly enhanced, called mutual extinction, or greatly reduced, mutual transparency, by up to $100 \%$ of the usual single-beam extinction. In view of the surprisingly strong mutual extinction and transparency, we propose new experiments to observe mutual extinction and transparency, namely in two-beam experiments with either elastic and absorbing scatterers, in optical wavefront shaping, in dynamic light scattering, and we discuss possible applications.
\end{abstract}

open laccess editor's choice Copyright (c) EPLA, 2020

Published by the EPLA under the terms of the Creative Commons Attribution 3.0 License (CC BY). Further distribution of this work must maintain attribution to the author(s) and the published article's title, journal citation, and DOI.

Introduction. - In many branches of physics, Nature is interrogated by performing scattering experiments [1]. An incident wave or particle beam impinges on a target sample, and characteristics of the scattered waves or particles are analyzed to find detailed information about the target. The theory of scattering is a mature, unifying branch of theoretical physics [2-4]. The formalism for the scattering of particles and the formalism for the scattering of waves are remarkably similar, despite the vast differences in their character, and the large varieties of particles and waves. In all treatises on scattering theory, the $S$-matrix and the $T$-matrix are key concepts to describe the target's influence on the scattering.

In traditional scattering experiments, a single beam of waves is incident on a target. Scattering experiments for classical waves, such as light, microwaves and sound, often involve the tremendous complication of multiple scattering that arises in turbid samples. Fortunately, much progress has meanwhile been made in understanding multiple scattering of classical waves [5-7]. In the recently developed popular method of wavefront shaping (WFS) [8,9], multiple beams are simultaneously incident on a turbid sample, with a range of incoming angles, amplitudes and phases. WFS has led to breakthroughs in imaging and focusing in turbid media. Right after the discovery of WFS in our group [10,11], many WFS experiments have been performed [12], even in the (ultrafast) time domain [13-15].

Theoretical efforts have been made to understand WFS in terms of the correlations in the transmitted light, see, for example, refs. [16-18]. In addition studies were made 
of advanced algorithms used to find the optimal wavefront, e.g., in refs. $[19,20]$. In our desire to understand more of the basic principles of WFS, we have recently embarked on calculating exactly the light scattering properties of a large number of dipoles acted on by a multiple of incoming waves. Our method to calculate the $T$-matrix of a set of dipoles is summarized in the appendix. In the WFS calculations we optimize the phases of all incoming beams to obtain an increased intensity in a single chosen outgoing direction. Our initial results were puzzling: it appeared as if energy was not conserved, although the optical theorem for the individual beams was obeyed.

Therefore, we decided to study in more detail the principles of the energy balance. By requiring the extinguished and scattered power to be equal for elastic scattering, independent of the amplitudes and phases of all incoming beams, we obtain a generalized optical theorem that appears to be known in abstract form [21] and in more detail for spherical scattering objects [2]. Our alternative derivation clarifies that when multiple beams are incident, there are cross-terms in the extinction and - most strikingly - that these cross-terms are experimentally observable. When incorporating the cross-terms, that we will refer to as mutual extinction, into our WFS calculations we find energy conservation to a very high precision, typically better than $10^{-16}$ relative error.

The occurrence of mutual interference in the extinction is important for interpreting scattering experiments with multiple incoming beams and points toward a number of new experiments. The simplest possible experiments where the mutual extinction will show up is in experiments with two beams. We suggest in this paper a number of two-beam experiments that will exhibit these mutual extinction effects. The beauty of these new and large mutual extinction effects is that they not only appear for white paint-like samples - that contain elastic scatterers with high albedo- but also for black paint-like samples that contain strongly absorbing particles with corresponding small albedo.

Theory. - In the scattering of light by multiplescattering objects to a large extent polarization effects are lost. So we will limit ourselves to scalar waves, although the extension of the theory to include polarization is straightforward.

In scattering theory it is appropriate to partition the field amplitude $\psi$ into an unperturbed part and a scattered part:

$$
\psi=\psi_{\text {in }}+\psi_{\text {scat }},
$$

making immediately clear that absorption is also a form of scattering. Extinction is the interference of the incoming beam with its own scattered beam, described by $\operatorname{Re}\left(\psi_{\text {in }} \psi_{\text {scat }}^{*}\right)$, whereas the scattered intensity is described by $\psi_{\text {scat }} \psi_{\text {scat }}^{*}$.

For far-field observations a more relevant quantity than the intensity is the current, for scalar waves defined as [22]

$$
\boldsymbol{J} \equiv-\operatorname{Re}\left[\left(\partial_{t} \psi\right)^{*} \nabla \psi\right]
$$

From the current $\boldsymbol{J}(\boldsymbol{r})$ the observable power $S$ can be calculated, $S=\lim _{r \rightarrow \infty} r^{2} \int \mathrm{d} \hat{\boldsymbol{r}} J_{r}$. We will denote the extinguished power as $S_{\text {ext }}$ and the scattered power as $S_{\text {scat }}$. When the scattering is elastic, $S_{\text {ext }}=S_{\text {scat }}$.

One incoming wave. We will start with the case of one incoming plane wave that is scattered. The amplitude in far field is given by

$$
\begin{aligned}
\lim _{r \rightarrow \infty} \psi(\mathbf{r})= & A e^{i \frac{\omega}{c} \hat{\boldsymbol{k}}_{\mathrm{in}} \cdot \boldsymbol{r}-i \omega t} e^{i \phi} \\
& +A f\left(\frac{\omega}{c} \hat{\boldsymbol{r}}, \frac{\omega}{c} \hat{\boldsymbol{k}}_{\mathrm{in}}\right) \frac{e^{i \frac{\omega}{c} r-i \omega t}}{r} e^{i \phi},
\end{aligned}
$$

where the real-valued $A$ represents the amplitude (units are the square root of the energy density), $\omega / c$ the magnitude of the wave vector, $\hat{\boldsymbol{k}}_{\text {in }}$ the incoming direction and $\phi$ the phase of the plane wave. The scattering amplitude $f\left(\frac{\omega}{c} \hat{\boldsymbol{k}}_{\text {out }}, \frac{\omega}{c} \hat{\boldsymbol{k}}_{\text {in }}\right)$, introduced in eq. (3), denotes the scattering strength from incoming direction $\hat{\boldsymbol{k}}_{\text {in }}$ to outgoing direction $\hat{\boldsymbol{k}}_{\text {out }}$. An alternative property to quantify scattering is the $T$-matrix, defined by $T\left(\frac{\omega}{c} \hat{\boldsymbol{k}}_{\text {out }}, \frac{\omega}{c} \hat{\boldsymbol{k}}_{\text {in }}\right) \equiv$ $-4 \pi f\left(\frac{\omega}{c} \hat{\boldsymbol{k}}_{\text {out }}, \frac{\omega}{c} \hat{\boldsymbol{k}}_{\mathrm{in}}\right)$. For one incoming wave the scattered power is the differential scattering integrated over solid angle,

$$
S_{\text {scat }}=\frac{\omega^{2}}{c} A^{2} \int \mathrm{d} \hat{\boldsymbol{k}}_{\text {out }}\left|f\left(\frac{\omega}{c} \hat{\boldsymbol{k}}_{\text {out }}, \frac{\omega}{c} \hat{\boldsymbol{k}}_{\text {in }}\right)\right|^{2},
$$

and the extinguished power becomes in this case $S_{\text {ext }}=$ $4 \pi \omega A^{2} \operatorname{Im} f\left(\frac{\omega}{c} \hat{\boldsymbol{k}}_{\mathrm{in}}, \frac{\omega}{c} \hat{\boldsymbol{k}}_{\mathrm{in}}\right)$. For extinction only the term $\hat{\boldsymbol{k}}_{\text {out }}=\hat{\boldsymbol{k}}_{\text {in }}$ survives (forward scattering) as the ever increasing spatially frequency in the phase of the crossterms wipes out any other contribution in $\psi_{\text {in }} \psi_{\text {scat }}^{*}$ (see footnote ${ }^{1}$ ). In the case of elastic scattering $S_{\text {ext }}=S_{\text {scat }}$ resulting in the famous optical theorem

$$
4 \pi \omega \operatorname{Im} f\left(\frac{\omega}{c} \hat{\boldsymbol{k}}_{\text {in }}, \frac{\omega}{c} \hat{\boldsymbol{k}}_{\text {in }}\right)=\frac{\omega^{2}}{c} \int \mathrm{d} \hat{\boldsymbol{k}}_{\text {out }}\left|f\left(\frac{\omega}{c} \hat{\boldsymbol{k}}_{\text {out }}, \frac{\omega}{c} \hat{\boldsymbol{k}}_{\text {in }}\right)\right|^{2} .
$$

Multiple incoming waves. The case of multiple incoming waves is described by the following field amplitude:

$$
\psi_{\mathrm{in}}(\boldsymbol{r})=\sum_{i=1}^{M} A_{i} e^{i\left(\frac{\omega}{c} \hat{\boldsymbol{k}}_{\mathrm{in}, i} \cdot \boldsymbol{r}-\omega t\right)} e^{i \phi_{i}}
$$

where each beam has its own real-valued amplitude $A_{i}$, direction $\hat{\boldsymbol{k}}_{\mathrm{in}, i}$ and its own phase $\phi_{i}$. When this set of incoming waves is sent to a target, all waves are scattered. Let us look at the current. Given the general decomposition (1) for each incoming wave we find, for each pair of incoming waves $i$ and $j$, sixteen contributions to the

${ }^{1}$ To be mathematically more precise: the extinction contribution is proportional to $\delta\left(\hat{\boldsymbol{k}}_{\text {out }}-\hat{\boldsymbol{k}}_{\text {in }}\right)$. 
current: four incoming contributions, four scattered contributions and eight extinction contributions. After calculating the extinction contributions and the scattering contributions and requiring them to be equal in the case of elastic scattering, and requiring the equality to be independent of all amplitudes and all phases, we arrive at

$$
\begin{aligned}
& 2 \pi \omega f\left(\frac{\omega}{c} \hat{\boldsymbol{k}}_{1}, \frac{\omega}{c} \hat{\boldsymbol{k}}_{2}\right)-2 \pi \omega f^{*}\left(\frac{\omega}{c} \hat{\boldsymbol{k}}_{2}, \frac{\omega}{c} \hat{\boldsymbol{k}}_{1}\right)= \\
& i \frac{\omega^{2}}{c} \int \mathrm{d} \hat{\boldsymbol{r}} f\left(\frac{\omega}{c} \hat{\boldsymbol{r}}, \frac{\omega}{c} \hat{\boldsymbol{k}}_{1}\right) f^{*}\left(\frac{\omega}{c} \hat{\boldsymbol{r}}, \frac{\omega}{c} \hat{\boldsymbol{k}}_{2}\right) .
\end{aligned}
$$

We verified this generalized optical theorem for our collection of dipoles and found for elastic scattering an agreement better than $10^{-16}$ relative error. We have refrained from dividing the l.h.s. and r.h.s. of eq. (7) by $2 \pi \omega$ because as it stands now the l.h.s. and the r.h.s. represent, respectively, exactly the extinction and the scattering power, after having multiplied the l.h.s. and r.h.s. terms by the appropriate wave amplitudes and phase factors and taking the imaginary part.

Two waves: proposed experiments. - Let us discuss several experimental settings where the mutual extinction could be observed. The simplest, highly relevant realization is the situation where one incoming (laser) beam is split $50 \%-50 \%$ into two beams and then sent to a sample. The two experimental properties that can be adapted by the experimentalist are the relative phases of the two beams and the angle $\gamma$ between them. A simplified set-up of the experiment is sketched in fig. 1 where we suggest to use a scattering sample with optical density of about 0.3 to ensure that the two extinguished beams are clearly visible and easy to detect.

Figure 1(a) shows two beams incident from the left on a complex medium consisting of scatterers with a high albedo. One incident beam has a phase shifter which, with the phase $\phi$, can be tuned and the other beam has a modulator for phase-sensitive detection, see, e.g., ref. [23]. Both beams emanate from the right of the slab - after experiencing a certain extinction - before propagating to their respective detectors. The phase-sensitivity detection is necessary because there will be a lot scattered light present.

The beauty of mutual extinction is that it also exists for strongly absorbing samples. In fig. 1(b) we sketch an experiment with two beams that are incident from the left on a complex medium consisting of scatterers with a low albedo.

The minimum observable level of change in the transmitted intensity of coherent light in these experiments is determined by the Poisson statistics of photodetection, and in WFS experiments it is of the order of $1 / \eta$, where $\eta$ is the maximal level of intensity enhancement achieved [24]. The enhancement has recently been shown to reach values on the order of $10^{5}[25]$, and, as a result, intensity fluctuations on this level should be detectable in an optimized setting. In the next section we will show by calculation (a)

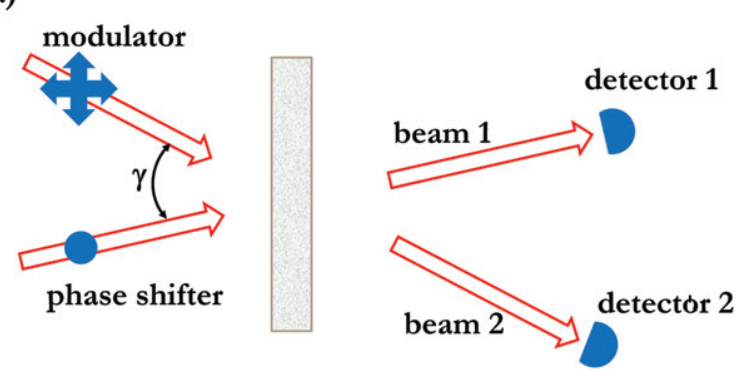

(b)

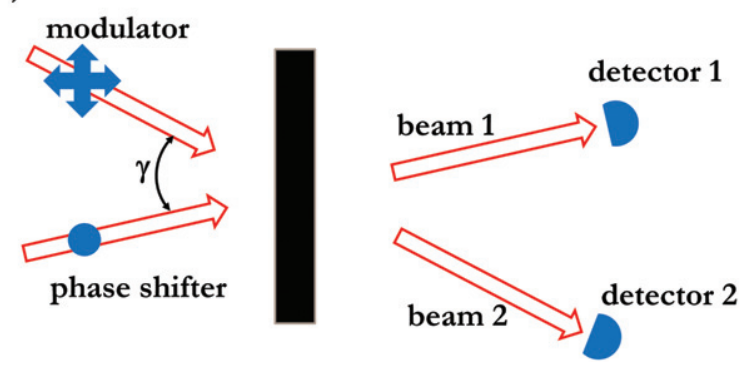

Fig. 1: (a) Schematic of an experiment in which two beams of equal intensity, derived from one laser beam, are incident on a semi-transparent white scattering slab. Phase-sensitive detection of the signal on beam 1 is completely due to mutual extinction. By tuning the phase shifter the mutual extinction can be varied between maximal and minimal for a particular angle $\gamma$. (b) Same as (a) with a semi-transparent absorbing sample. This experiment will prove that mutual extinction also occurs in the case of absorption only, i.e., in the absence of scattered intensity.

that mutual extinction is so large that in the case of the experiment sketched in fig. 1(b), it might even be visible to the naked eye as in that case there is no stray light.

Magnitude of mutual extinction. - To assess the importance of mutual extinction, its magnitude is paramount. Therefore, we have calculated the mutual extinction for large samples consisting of many scatterers by exact evaluation of their $T$-matrix (see appendix). Moreover, we have estimated the magnitude of mutual extinction by applying Fraunhofer diffraction theory (FDF) $[26,27]$ that is asymptotically correct the more opaque the scattering object is and the larger its size is in comparison to the wavelength.

Figure 2 shows the total extinction as a function of the phase difference between the two beams that are incident on a large slab-like box that contains no less than $10^{4}$ scattering particles. For a phase difference $\phi=0$ the total extinction is strongly reduced, indicating mutual transparency, and for $\phi=\pi$ the total extinction is strongly increased, signalling mutual extinction. The total extinction shows a smooth sine interference pattern with an amplitude of $25 \%$ that is readily observable in an experiment.

Figure 3 shows the maximum and minimum total extinction obtained from interference patterns as in fig. 2, as 


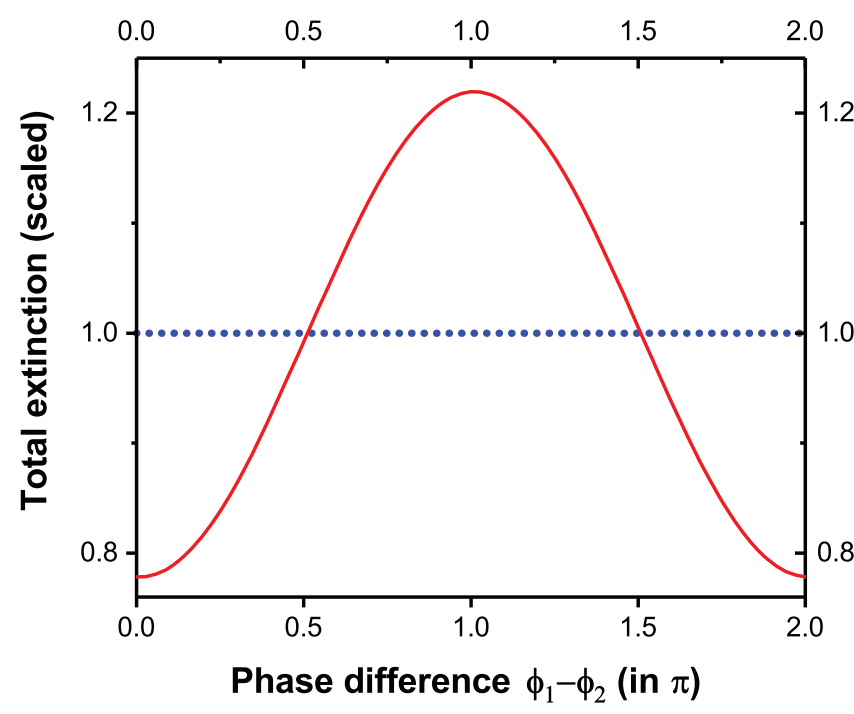

Fig. 2: Calculated total extinction (red curve) in a two-beam experiment as shown in fig. 1(a) as a function of the phase difference $\left(\phi_{1}-\phi_{2}\right)$ between the two beams. The scattering sample is a rectangular box of size $80 \lambda \times 80 \lambda \times 10 \lambda$ containing 10000 randomly positioned elastic point dipoles on resonance. The mean free path is $\ell=20.1 \lambda$, and the angle between the two beams is $\gamma=1^{\circ}$. The extinction with only the sum of the forward scattering (self-extinction) of the two beams included, is the horizontal blue dotted line. Both graphs are scaled by the sum of the forward scattering of the beams at zero angle.

a function of the angle between the two incident beams. In the limit of small angles, the mutual extinction is extremely strong, with modulations up to $100 \%$, as shown in fig. 3(a). At about $\gamma=0.75^{\circ}$ the mutual extinction vanishes, before increasing again to $25 \%$ at $\gamma=1^{\circ}$ (cf. fig. 2). Since the range between $0.75^{\circ}$ and $1.4^{\circ}$ is the first speckle spot, it is obvious that the large mutual extinction is transferred by the scatterers. Figure 3(b) shows that at much larger angles the mutual extinction is less but still at an experimentally observable few percent level. The pattern is more speckle-like than at small angles, probably since we are probing large-wave-vector effects of the sample.

For the diffraction model we assume that the form of the scattering object is a 3D rectangular box with as $x$-size $a$, as $y$-size $b$ and as $z$-size $c$. We begin with a rectangular slit lying in the $z=0$ plane with the same $x$-size and $y$-size as the box. After calculating the diffraction properties of this slit we use Babinet's principle to infer the scattering properties of the box, just by reversing the sign of the diffracted amplitude. Consider a beam incident on this 2D rectangular slit, with the incident direction $\hat{\boldsymbol{k}}_{\text {in }}$ defined by its three direction cosines $\left\{\cos \theta_{x, \text { in }}, \cos \theta_{y, \text { in }}, \cos \theta_{z, \text { in }}\right\}$ and with the outgoing direction $\hat{\boldsymbol{k}}_{\text {out }}$, defined by its three direction cosines $\left\{\cos \theta_{x, \text { out }}, \cos \theta_{y, \text { out }}, \cos \theta_{z \text {,out }}\right\}$. With Fraunhofer diffraction (FDF) theory one can easily calculate the amplitude for outgoing direction $\hat{\boldsymbol{k}}_{\text {out }}$ and incoming direction $\hat{\boldsymbol{k}}_{\text {in }}[26,27]$. By reversing the sign of this amplitude and adding the incoming beam to the result, the total
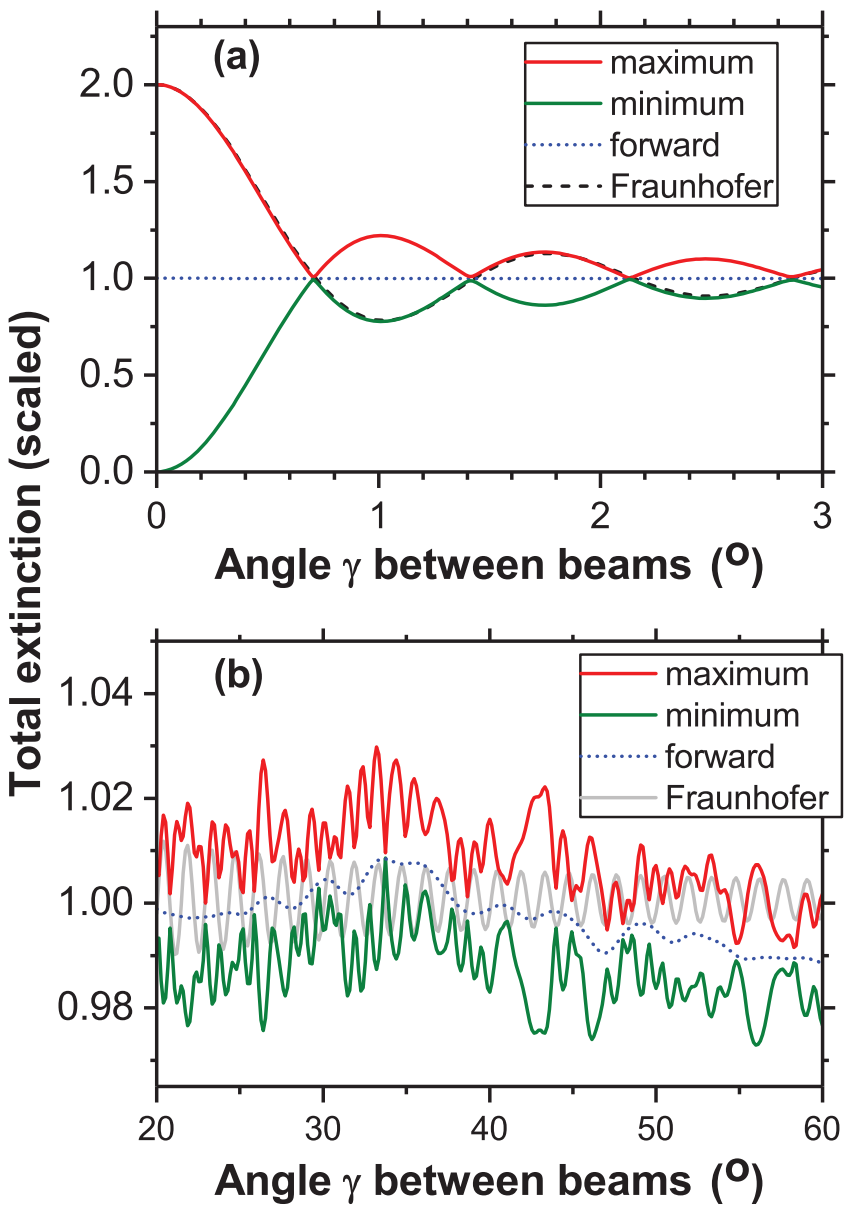

Fig. 3: (a) Calculated total extinction in a two-beam experiment as shown in fig. 1(a) as a function of the angle $\gamma$ between the two beams. At each angle the relative phase was varied to obtain the maximal (green curve) and the minimal (red curve) mutual extinction, see fig. 2. The properties of the scattering object and the extinction scaling are given in the caption of fig. 2. The result of Fraunhofer diffraction theory (black dashed curve) agrees very well with the exact results if a multiplicative normalization factor of 0.22 is used. The extinction with only the sum of the forward scattering (self-extinction) of the two beams included, is the blue dotted line. (b) Same sample as (a) for large angle $\gamma$ between the beams. Mutual extinction reveals itself as a speckle for all angles, with a magnitude of a few percent. Here the Fraunhofer result (grey curve) differs, as expected.

diffracted amplitude of the rectangle is of the standard form (4) with

$$
f_{\mathrm{FDF}}\left(\frac{\omega}{c} \hat{\boldsymbol{k}}_{\text {out }}, \frac{\omega}{c} \boldsymbol{k}_{\mathrm{in}}\right)=\frac{i a b}{\lambda} \operatorname{sinc}(\alpha) \operatorname{sinc}(\beta),
$$

where $\alpha \equiv \frac{\omega}{2 c} a\left(\cos \theta_{x, \text { out }}-\cos \theta_{x, \text { in }}\right)$ and in which $\beta \equiv \frac{\omega}{2 c} b\left(\cos \theta_{y, \text { out }}-\cos \theta_{y, \text { in }}\right)$. By writing the Fraunhofer diffraction result in form (8) we have generalized Fraunhofer diffraction to account for mutual extinction. Formula (8) shows that the mutual extinction can be huge, of the same order as the self-extinction. A fact we already discovered from our exact numerical calculations. 
Fraunhofer diffraction is based on the scattering sample being impenetrable. We introduce a multiplicative normalization factor to bring at zero angle the extinction calculated with diffraction theory exactly equal to the rigorous result. We have found, as expected, that the smaller the mean-free path gets, the closer the normalization factor approaches 1 . For instance for a block of $8 \lambda \times 8 \lambda \times 1 \lambda$ containing 1000 dipoles the mean free path is $0.20 \lambda$ and the normalization factor for this nearly completely opaque sample is 0.97 .

In the more translucent sample of fig. 3 we see that the result from Fraunhofer diffraction is in excellent agreement with the exact theory at small angles when using 0.22 as the multiplicative normalization factor. Conversely, at large angles diffraction theory gives poor results, see fig. 3(b), as expected: Fraunhofer diffraction is based on the scattering object being impenetrable and the impenetrability of opaque samples becomes less with oblique incidence.

In our theory the incoming beams are plane waves, whereas in an experiment one has to deal with beams of finite extent leading to an overlap factor that will reduce the signal compared to the theoretical result.

\section{Generalizations. -}

Wavefront shaping. The results in this paper suggest to perform WFS experiments where the merit function concerns the minimizing or maximizing of the extinction. The concept of mutual extinction is generalized in such experiments from two incident beams to a large number of, individually phase-adjustable, incident modes. The extinction of one beam, or a linear combination of many incident beams, could be maximized or minimized by optimizing the phases of the incident waves, either using direct optimization methods or methods derived from linear algebra [28].

In several papers, the intriguing possibility of maximizing or minimizing total transmission, reflection, or absorption was explored both theoretically and experimentally $[9,11,29-31]$. We note that while these quantities possibly correlate with extinction, these papers consider intensities summed over many modes, whereas our new insights into mutual extinction concern the power lost from or added to each individual incident mode.

Polarization. Up to now we only considered light to be well described by scalar waves. And indeed the extension to vector waves is not to be expected to bring about exciting new features. With one exception: the two-beam experiment with orthogonal polarizations. From eq. (8) and from fig. 3 we notice that the mutual extinction for scalar waves becomes maximal when the two beams almost overlap. This would not be the case if the two beams would have crossed polarizations. When those two beams overlap the mutual extinction would be zero and would rapidly increase on enlarging the angle between the two beams. At larger angles the results will be the same as with the beams having identical polarizations.
Dynamic light extinction. A very popular optical technique in physics, chemistry, biology, and in industry is dynamic light scattering (DLS) [32]. In DLS one uses samples where the concentration of scatterers that are moving is so low that on average the observed scattered intensity has only scattered once when it leaves the sample. The time correlation of that scattered intensity gives valuable information on the motion of the scattering particles. For opaque samples the technique of Diffuse Wave Spectroscopy was invented [33,34]. Here we suggest to introduce Dynamic Light Extinction by measuring with phase-sensitive detection the dynamic correlation function $\left\langle I_{1}(0) I_{1}(t)\right\rangle$, where $I_{1}$ is the intensity of beam 1 measured by detector 1 as sketched in fig. 1. The sample would contain moving elastic scatterers (see fig. 1(a)) or moving absorbing particles (see fig. 1(b)). Observation of the motion of absorbing particles by optical means would open up a lot of applications in the sciences and in industry.

Conclusions. - On the basis of exact cluster calculations we have shown that with multiple light beam incidence there is mutual extinction and that its magnitude is substantial and observable. Based on these exact computations of clusters containing many scatterers and borne out by Fraunhofer diffraction theory we make the striking observation that the mutual extinction and mutual transparency of two incident waves is as large as $100 \%$ of the self-extinction. For small angles between the beams the mutual extinction is large (up to 100\%) and very well described by diffraction theory. For large angles the mutual extinction is smaller, at the percent level, but still observable. At large angles the mutual extinction is speckle-like and poorly described by diffraction theory.

In view of the surprisingly strong mutual extinction and mutual transparency, we have proposed several new experiments to observe mutual extinction, namely in twobeam experiments, in optical wavefront shaping, in dynamic light scattering, and discuss possible applications.

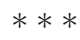

We thank LARS CORBIJN VAN WILlEnswaARD for advice on the code, and Bill Barnes, Pepijn Pinkse, and IVO VELLEKOOP for discussions on wavefront shaping. We acknowledge support by NWO-TTW Perspectief program "Free-form scattering optics" and NWO-Vici 68047618.

Appendix: exact calculation of $T$-matrix. - The complex scattering medium is taken to consist of a collection of $N$ scatterers. Scatterers are labeled as $\alpha, \beta, \gamma, \delta$, etc. and the location of scatterer $\alpha$ is represented as $\mathbf{R}_{\alpha}$. The scattering of a point scatterer is characterized by its single particle $t$-matrix $t[35]$.

We will calculate the $T$-matrix of the whole scattering object by summing the scattering events order by order, up to infinite order. As the $t$-matrix of an individual scatterer sums up all interactions with that scatterer, we should exclude double scattering from that same scatterer. 
When multiple summations over the scatterers are performed, we would rather have $\sum_{\alpha} \sum_{\beta}$ than $\sum_{\alpha \neq \beta} \sum_{\beta}$. These awkward conditions on the indices can be avoided by defining the matrix $\boldsymbol{D}$,

$$
D_{\alpha \beta} \equiv\left(1-\delta_{\alpha \beta}\right) G_{\alpha \beta},
$$

where $G_{\alpha \beta} \equiv G\left(\left|\mathbf{R}_{\alpha}-\mathbf{R}_{\beta}\right|\right)$, the Green's function for scalar waves in homogeneous space. By replacing $\boldsymbol{G}$ by $\boldsymbol{D}$ in our summations, double counting of scattering events off the same scatterer is automatically excluded in the summations, without having to explicitly exclude them. In addition we define the matrix $\tau$ by $\tau_{\alpha \beta} \equiv \delta_{\alpha \beta} t_{\alpha}$.

Let us consider, for instance, the contribution of secondorder scattering, which is given by

$$
T_{2}\left(\mathbf{r}_{1}, \mathbf{r}_{2}\right)=\sum_{\alpha, \beta} \delta\left(\mathbf{r}_{1}-\mathbf{R}_{\alpha}\right) \delta\left(\mathbf{r}_{2}-\mathbf{R}_{\beta}\right) t_{\alpha}(\boldsymbol{D} \boldsymbol{\tau})_{\alpha \beta} .
$$

Similar expressions can be found for higher orders and the resulting geometric series can be summed exactly into

$$
T\left(\mathbf{r}_{1}, \mathbf{r}_{2}\right)=\sum_{\alpha, \beta} \delta\left(\mathbf{r}_{1}-\mathbf{R}_{\alpha}\right) \delta\left(\mathbf{r}_{2}-\mathbf{R}_{\beta}\right) T_{\alpha \beta},
$$

where $T_{\alpha \beta}$ is defined as

$$
T_{\alpha \beta} \equiv t_{\alpha}[\boldsymbol{I}-\boldsymbol{D} \boldsymbol{\tau}]_{\alpha \beta}^{-1} .
$$

To calculate the full $T$-matrix $T\left(\mathbf{r}_{1}, \mathbf{r}_{2}\right)$, and so all the scattering properties of the collection of $N$ point dipoles, we have to invert a $N \times N$ matrix. In practice this can be done without any special computational requirements for up to 10000 point dipoles. In contrast to the popular Foldy-Lax method [36,37], our method directly gives the $T$-matrix of the scattering object, but the two methods can be shown essentially to be equivalent.

\section{REFERENCES}

[1] LAndau L. D. and Lifshitz L. M., Quantum Mechanics: Non-Relativistic Theory, 2nd edition (Pergamon, London) 1965.

[2] Newton R. G., Scattering Theory of Waves and Particles, 2nd edition (Springer-Verlag, Berlin) 1982.

[3] Joachain C. J., Quantum Collision Theory (NorthHolland, Amsterdam) 1983.

[4] TAYLOR J. R., Scattering Theory: The Quantum Theory of Nonrelativistic Collisions (Dover, Mineola, NY) 2006.

[5] Van Rossum M. C. W. and Niewenhuizen T. M. N., Rev. Mod. Phys., 71 (1999) 313.

[6] Akkermans E. and Montambaux G., Mesoscopic Physics of Electrons and Photons (Cambridge University Press, Cambridge, UK) 2007.

[7] Wiersma D. S., Nat. Photon., 7 (2013) 188.

[8] Mosk A. P., LagendiJk A., Lerosey G. and Fink M., Nat. Photon., 6 (2012) 283.
[9] Rotter S. and Gigan S., Rev. Mod. Phys., 89 (2017) 015005.

[10] Vellekoop I. M. and Mosk A. P., Opt. Lett., 32 (2007) 2309.

[11] Vellekoop I. M. and Mosk A. P., Phys. Rev. Lett., 101 (2008) 120601.

[12] Kubby J., Gigan S. and Cui M. (Editors), Wavefront Shaping for Biomedical Imaging (Cambridge University Press, Cambridge, UK) 2019.

[13] Katz O., Small E., Bromberg Y. and Silberberg Y., Nat. Photon, 5 (2011) 372.

[14] Aulbach J., Guonaj B., Johnson P. M., Mosk A. P. and LagendiJk A., Phys. Rev. Lett., 106 (2011) 103901.

[15] McCabe D. J., Tajalli A., Austin D. R., Bondareff P., Walmsley I. A., Gigan S. and Chatel B., Nat. Commun., 3 (2011) 447.

[16] Shapiro E. A., Drane T. M. and Milner V., Phys. Rev. A, 84 (2011) 053807.

[17] Davy M., Shi Z. and Genack A. Z., Phys. Rev. B, 85 (2012) 035105.

[18] Hsu C. W., Liew S. F., Goetschy A., CaO H. and Stone A. D., Nat. Phys., 13 (2017) 497.

[19] Horisaki R., Takagi R. and Tanida J., Appl. Opt., 56 (2017) 4358.

[20] Fayyaz Z., Mohammadian N., Salimi F., Fatima A., Reza Rahimi Tabar M. M. R. R. and Avanaki M. R. N., Appl. Opt., 57 (2018) 6233.

[21] Messiah A., Quantum Mechanics, Vol. II (NorthHolland, Amsterdam) 1961.

[22] LagendiJk A. and van Tiggelen B. A., Phys. Rep., 270 (1996) 143.

[23] Demtröder W., Laser Spectroscopy, Vol. 1: Basic Principles (Springer-Verlag, Berlin, Heidelberg) 2008.

[24] Yilmaz H., Vos W. L. and Mosk A. P., Biomed. Opt. Express, 4 (2013) 1759.

[25] Yu H. S., LeE K. R. and PARK Y. K., Opt. Express, 25 (2017) 8036.

[26] Born M. and Wolf E., Principles of Optics, 7th edition (Cambridge University Press, Cambridge, UK) 2019.

[27] Неснт E., Optics, Global Edition (Pearson Education, London, UK) 2016.

[28] Popoff S. M., Lerosey G., Carminati R., Fink M., Boccara A. C. and Gigan S., Phys. Rev. Lett., 104 (2010) 100601.

[29] Chong Y. D., Ge L., Cao H. and Stone A. D., Phys. Rev. Lett., 105 (2010) 053901.

[30] Gérardin B., Laurent J, Derode A., Prada C. and Aubry A., Phys. Rev. Lett., 113 (2014) 173091.

[31] Liew S. F. and Cao H., Opt. Express, 23 (2015) 11043.

[32] Berne B. J. and Pecora R., Dynamic Light Scattering: With Applications to Chemistry, Biology, and Physics (Dover, Mineola, NY) 2013.

[33] Maret G. and Wolf P. E., Z. Phys. B, 65 (1987) 409.

[34] Pine P. J., Weitz D. A., Chaikin P. M. and Herbolzheimer E., Phys. Rev. Lett., 60 (1988) 1134.

[35] de Vries P., van Coevorden D. V. and LagendiJk A., Rev. Mod. Phys., 70 (1998) 447.

[36] Foldy L. L., Phys. Rev., 67 (1945) 107.

[37] Lax M., Rev. Mod. Phys., 23 (1951) 287. 